\title{
Entre la proscripción y la construcción de una identidad: el peronismo universitario a inicios de los sesenta
}

Between proscription and the building of an identity: peronism in university in the early sixties

\author{
Julián Andrés Dércoli jdercoli@gmail.com \\ https://orcid.org/0000-0002-8746-1226
}

Universidad Nacional Arturo Jauretche (Argentina)

\section{Resumen}

La relación entre el peronismo y la universidad fue estudiada a partir de un conjunto de prejuicios que afirmaban su total antagonismo. Con el fin de poner en tela de juicio a los 
mismos, analizaremos el discurso sobre la universidad de la revista 4161; la misma se autodenominada órgano de la Juventud Universitaria Peronista y sus dos números aparecieron entre 1963 y 1964. A nuestro juicio, la relevancia de la revista radica en que fue una aparición temprana de un conjunto de universitarios dados a la tarea de construir una identidad universitaria peronista. La idea que guía nuestra pesquisa es que la revista ensaya un sincretismo que, partiendo del peronismo, busca converger con diversas corrientes como el reformismo o el marxismo. Este proceso es de vital importancia para dar cuenta de la posterior masividad que adquiere el peronismo en las universidades en la década del 70 y, hacia 1973 y cómo aquellas concepciones influyeron en los proyectos de reforma institucional de las universidades argentinas. Por este sendero, podemos pensar que 4161 fue el inicio de una cultura política universitaria del peronismo.

Palabras clave: Peronismo; universidad; 4161; JUP; identidad.

\section{Abstract}

The relationship between Peronism and University has been studied on the basis of the prejudice that upheld their mutual antagonism. With de purpose of challenging this prejudice, we shall analyse the discourse about university in 4161 magazine. 4161 designated itself as the news media of the University Peronist Youth (JUP for its acronym in Spanish), and its two issues were released between 1963 and 1964. From our point of view, the relevance of this magazine is based on the fact that it meant the early appearance of a group of university students aiming at building a peronist university identity. The leading idea of our research is that the magazine attempts at a syncretism that, based on peronism, looks at converging with other identities, such as reformism or marxism. This process is crucial to understand the subsequent popularity that peronism gains in the university world in the seventies and towards 1973, as well as its influence in the projects of institutional reform during this period. Along the same lines, we might affirm that 4161 meant the beginning of the university political culture of peronism.

Keywords: Peronism; university; 4161; JUP; identity.

En los años setenta el peronismo había logrado una influencia considerable en el ámbito universitario, si tenemos en cuenta que durante el primer peronismo sectores mayoritarios de los universitarios habían sido férreos opositores ¿Cómo podemos entender este cambio de 
posiciones? Un conjunto de trabajos (Cfr. Terán, 2013; Altamira, 2013) abordaron este proceso de cambios desde una perspectiva general. Dentro de este proceso debe incluirse lo que ocurrió en la Universidad, pero rescatando la particularidad que, a nuestro juicio, constituye que un conjunto de lineamientos que orientaron la acción de la política universitaria del peronismo en los sesentas impactaron en la transformación institucional de las universidades. Muchos de eso lineamientos ya se encuentran sostenidos como diagnósticos y, forman parte central, de lo que aquí llamamos la construcción de una identidad por parte del peronismo universitario. Estudiaremos este proceso a partir del análisis de la revista 4161, órgano de la Juventud Universitaria Peronista, que surgida en el 1963 ya perfila cuestiones centrales de lo que unos diez años más tarde tendrá una institucionalización en la Universidad Nacional y Popular de Buenos Aires o en la Ley Taiana. Lo cual nos estaría dando indicios de un proceso de constitución de una cultura política universitaria del peronismo que actúa como hilo que une estas experiencias.

Nuestro objetivo es reconstruir algunos rasgos específicos de aquella identidad política: sus particularidades y sus diferencias con las tradiciones universitarias hegemónicas, la articulación entre militantes con intelectuales del "campo nacional" y la articulación de un discurso sobre la universidad con la historia de un movimiento político al que muchos universitarios, con algunas excepciones, habían calificado como autoritario.

\section{Breve estado de la cuestión y aspectos metodológicos}

Un conjunto de trabajos que abordaron el proceso de politización de los intelectuales y universitarios en la Argentina de la década del sesenta. Algunos centraron su atención en los acercamientos entre nacionalismo y marxismo como rasgo característico de ese proceso de politización (Terán, 2013). Otros, abordaron el vínculo entre el peronismo y una denominada cultura de izquierda, como lo hiciera Altamirano (2013). Según el producto de este cruce se produjeron actualizaciones teóricas-políticas para el peronismo, en clave anti-imperialista y de corte clasista, y para la izquierda el acercamiento a un concepto de lo popular y nacional para pensar y actuar en la política. A lo que se suma el de una cultura política marcada por el antiintelectualismo, la demonización de la clase media y la auto-culpabilización de estos intelectuales-militantes por su origen de clase, lo que afirmará ciertos tópicos críticos de dicho sector como clave generacional

En otro andarivel encontramos el trabajo de Neiburg Los intelectuales y la invención el peronismo (1998), estudio que concentra su visión en los años posteriores al golpe de 1955. 
Aquí aparecen algunos elementos que interesan a nuestro estudio, tales como los sentidos que adquiere la Reforma en estos procesos de institucionalización académica y de perspectiva de la universidad luego de 1955. Lo distintivo del autor es el abordaje de la relación peronismo e intelectuales a través del concepto de invención.

Un trabajo clásico es el libro de Sigal (2002) Intelectuales y poder en Argentina, concentrado en las transformaciones en el campo universitario. Ella nos introduce, por un lado, en las representaciones construidas en torno a la relación modernización, reforma, desarrollismo y, por otro, intentando dar cuenta de los cambios en el campo intelectual en la década del sesenta, en clave de radicalización. Dichos elementos nos permiten abordar la problemática de la legitimación de la tarea intelectual y la producción cultural y su relación con el campo político. Otro conjunto de trabajos desarrollaron su interés en la relación entre los universitarios y el peronismo en el período que va de fines de los sesenta hasta el golpe de Estado de 1976 (Barletta, 2001; Friedemann, 2017; Dip, 2012; Recalde y Recalde, 2007). Más allá de sus distintas particularidades, estos trabajos podrían ser reunidos bajo el siguiente criterio: parten de que el peronismo tiene una acción sobre el campo universitario e intelectual propositiva que merece ser estudiada desde su especificidad.

Uno de los conceptos centrales es el cultura política, de acuerdo con Berstein (1997) esta es "una lectura común del pasado y una proyección hacia futuro vivida en grupo" (p. 392). También utilizaremos el concepto invención de la tradición (Hobsbawm, 1983). Aunque este concepto es utilizado para el estudio de las naciones, puede sernos útil para entender lo que a veces sin más se llama tradición reformista o universidad reformista. Para este análisis también consideramos necesario utilizar la categoría de tradición selectiva (Williams, 2009), de forma tal de observar los "recortes" realizados a la experiencia histórica y su funcionalidad en la construcción de la nueva identidad. Nos referimos a esto a la posibilidad de pensar al reformismo como un recorte de la Reforma, esto es una reinterpretación del movimiento y sus reivindicaciones a partir de la luz del presente de ese grupo. No podemos dejar de ver a 4161 bajo este mismo marco, ya que los editores-militantes enfrentan el desafío de tener que inventar una legitimidad para este peronismo universitario, que además debe construir una imagen de sus adversarios; a la vez que un recorte propio que intente una explicación de la antigua oposición al peronismo.

Por último, la revista 4161 se nos presenta como una fuente privilegiada para acceder a estos temas ya que como cualquier revista puede ser analizada desde una lectura socio-histórica, que la ubica como lugar de enunciación de problemas, de manifestación de discusiones, de afirmación de autores en un determinado campo (Sarlo, 1992; Faierman, 2017), es decir la revista como ese diálogo entre cultura y política.

Question, Vol. 1, N. ${ }^{\circ}$ 63, julio-septiembre 2019. ISSN 1669-6581

Instituto de Investigaciones en Comunicación | Facultad de Periodismo y Comunicación Social | Universidad Nacional de La Plata La Plata | Buenos Aires | Argentina

Página 4 de 18 


\section{Descripción de la revista}

4161 se autodenominaba el órgano de la Juventud Universitaria Peronista (JUP). Solamente tuvo dos números en formato papel: uno de ellos en 1963 y el otro en 1964, con 19 y 20 páginas respectivamente. Se trata de una temprana aparición de esta sigla que nucleaba a una serie de agrupaciones de diversas facultades de la Universidad de Buenos Aires. De acuerdo a lo mencionado por Hugo Chumbita (1), el Consejo de redacción tenía un funcionamiento horizontal y democrático, la mayoría de las notas salían sin firma lo cual indicaba que era la línea acordada por dicho organismo, mientras que las otras notas salían con las iniciales del autor como mecanismo de resguardo de su identidad.

En la revista podemos reconocer tres dimensiones: la primera refiere a la cuestión universitaria, la segunda se centra en la discusión sobre la coyuntura política argentina y, por último, la discusión en torno al Tercer Mundo, el imperialismo, la tercera posición y la polémica entre justicialismo y marxismo. Es menester destacar, que hay una dimensión que está por sobre las anteriores, digamos que organiza el sentido y que se presenta como transversal, nos referimos a la dimensión histórica en clave revisionista. El grupo encuentra en la política la actividad que da sentido a todas las demás, rasgo propio de aquel período (Terán, 2013: 47). Ahora bien, si la política es la que dota de sentido a toda acción presente, un rasgo particular es que la política es pensada en clave histórica. En repetidas ocasiones aparece la idea de que el presente es la repetición de un conflicto que viene de antaño y es aquel que estructura nuestra historia: o somos patria o permanecemos colonia. Otro vínculo entre política e historia en la revista, es aquel que conecta la correcta posición política y una supuesta razón de la historia. Esto aparece con claridad en el artículo Argentina y el Peronismo (4161, 1963: 1), allí luego de una cita a un mensaje de Perón se desarrolla la siguiente reflexión:

En contraste con la extraordinaria lucidez de estas palabras, que ubican nuestra estrategia en 'coincidencia con la naturaleza del desarrollo histórico', más de una vez hemos visto conducción táctica proceder con métodos que la realidad política que afrontamos ha vuelto inconducentes (4161, 1963: 4).

En este discurso política y razón de la historia se legitiman circularmente entre sí, haciendo claro el vínculo entre historia y legitimación de la política, en tanto esta estuviera acorde a la "naturaleza del desarrollo histórico".

Todos estos elementos aportan a la construcción de la identidad del grupo y la base sobre la cual se afirmará la política de la JUP para la universidad ya que, como veremos más adelante, 
esta institución será caracterizada tal como si estuviese atravesada por el mismo conflicto que la Nación. Lo cual lo deducimos de los artículos así como de la reseña de actividades militantes que hay en ambos números. Allí se daba cuenta de un conjunto de iniciativas que realizaba la organización. En el número 2 (1964: 19) se mencionan dos actividades de corte específicamente histórico: por un lado, una mesa redonda organizada por el aniversario de la batalla de la Vuelta de Obligado. Dicha actividad se llevó a cabo en la Facultad de Filosofía y Letras en la cual además de referentes de las agrupaciones hablaron José Baxter de Tacuara y Arturo Jauretche. Se suma también el informe en torno a la Comisión de Homenaje Permanente al Chacho Peñaloza. Según se relata en el primer número de la revista, a dicha Comisión adherían 13 agrupaciones universitarias entre ellas reformistas, humanistas, de izquierda nacional y peronistas. En el segundo número, se llevará adelante un racconto de las dificultades en términos ideológicos para que dicha comisión avance. También aparecen actividades en las que disertaron José María Rosa y Hernández Arregui, además de una reseña al libro de este último. Las actividades de formación y divulgación eran momentos de afirmación de una identidad; no sólo por el contenido sino porque muchas de ellas se llevan en locales sindicales, lo cual se reivindicaba como la afirmación del vínculo con el movimiento obrero, así como tomas de facultades en coordinación con medidas de fuerza de la CGT. También en estas partes de la revista se daba cuenta de la participación en elecciones estudiantiles, en elecciones nacionales, en, entre otras.

La JUP construye su identidad a partir de ese anclaje histórico en el movimiento popular y, al mismo tiempo, buscaba construir una identidad con una especificidad universitaria; por eso, los artículos insisten en superar las concepciones de "otros grupos estudiantiles [que] agotan su 'función social' y su 'compromiso' en las aulas, en las puertas o aún en los inoperantes institutos de extensión universitaria, nuestra acción se ha ligado concretamente con los problemas, movilizaciones y organizaciones gremiales del pueblo trabajador" $(4161,1963)$. En otros términos, la JUP plantea la disputa de la universidad y sin renegar del lugar de universitarios; este aspecto aparece en la primera editorial: "Los universitarios peronistas expresamos en estas páginas una actitud ante los problemas del país y del mundo, desde nuestra perspectiva nacional, popular e hispanoamericana" (4161, 1963: 1).

Otro aspecto que aparece con cierta recurrencia es la crítica al comportamiento político de las clases medias. Algunas veces esto es usado para describir la ambivalencia de los universitarios, otras para dar cuenta de la coyuntura, por ejemplo, la revista afirmó que "el miedo y el papel ambiguo y vacilante, que juegan las clases medias en la política argentina" (4161, 1963: 7) llevó al triunfo de Illia que "parece haber interpretado momentáneamente las ansias de seguridad de esa parte del pueblo" (7). Dichas críticas nos remiten al planteo de 
Altamirano (2013), quien afirma como elemento característico del cruce de peronismo y cultura de izquierda la crítica a las clases medias, que él sintetiza con un título: la clase media, una clase en el purgatorio. Sin embargo, no encontramos en la revista una estructura de autoculpabilización como motivo para explicar el "paso" hacia una identidad peronista. Destacamos esta ausencia, dado que en el trabajo de Altamirano esto aparece como un elemento central para pensar la relación entre los universitarios y lo popular.

Continuemos, otro rasgo que aparece con fuerza es el papel de la juventud como "una esperanza cierta", se habla de una juventud madura que se deducía de su grado organizativo, "como factor dinámico de renovación y superación" (4161, 1963: 9); según la revista, aquella era también "expresión insobornable de la rebeldía de las nuevas generaciones" (7). Tal vez, la idea de una juventud madura, pueda ligarse a la necesidad de dejar de concebirla como una edad pequeño-burguesa. Más allá de esto, lo cierto es que se daba un lugar de importancia a la Juventud Peronista en el proceso político argentino, pero "sin preeminencias de unos [sectores] sobre otros" (7). Perspectiva que se traslada como esquema para pensar la política en la universidad: los universitarios eran un sector importante pero que debía estar junto a los trabajadores. Dicho planteo se llevó a la práctica mediante instancias de formación conjuntas y de coordinación de acciones con sectores del sindicalismo; perspectiva que nos parece una formulación original, ya que no renegaba de la lucha específicamente universitaria, pero la situaba en sintonía con una estrategia política nacional.

Para finalizar, resta decir que los diversos artículos sostienen que el peronismo universitario es el único actor capaz de encarar la batalla por una universidad nacional, debido a que es el movimiento nacional que encarna la lucha por la liberación, razón la cual es capaz de articular la lucha universitaria en una perspectiva más amplia. Desde este esquema se articulaba un escenario dicotómico que permitía que la incorporación del peronismo a la disputa universitaria; dado que este traía consigo una historia de democratización de la universidad, como fue el desarancelamiento durante el primero peronismo y de desarrollo de un conocimiento soberano, como lo venía haciendo el revisionismo a partir de su combate con la política de la historia de la oligarquía. A nuestro juicio, estos son elementos centrales, que usa la revista 4161, para configurar una cultura política del peronismo universitario, la cual será el sustento para la militancia de la JUP.

\section{¿Hacia una cultura política universitaria del peronismo?}

En los años posteriores al golpe de Estado de 1955 los universitarios, intelectuales y sus revistas tuvieron una absoluta preponderancia en la construcción de las interpretaciones en 
torno al "hecho peronista". Luego muchos revisaron dichas posiciones terminaron por afirmar una visión más positiva del peronismo. Pero al principio existía un amplio consenso en la lectura del golpe de Estado como un momento de triunfo de la libertad por sobre el autoritarismo. En la universidad esto se tradujo en la construcción de una representación y un imaginario particular: mientras las mayorías eran proscriptas aquel momento fue vivido como el retorno a los principios de la Reforma. Más tarde, será identificado con la idea de "la edad de oro" para describir lo que ocurría en la universidad y con la investigación científico-tecnológica (Buchbinder, 2011). Estas interpretaciones forjaban un sentido opuesto a la etapa peronista, que era signada con la idea de "oscuridad". En función de esto, la pregunta que surge es la siguiente: ¿Por qué hacia 1973 el ámbito universitario ya no prestará tan aguda oposición al peronismo? Es más, con el retorno del peronismo al poder, se abrió un escenario de reformas institucionales, por ejemplo, el proceso de la Universidad Nacional y Popular de Buenos Aires y la sanción de una nueva Ley Universitaria; ambos procesos son profundamente críticos del período pos Revolución Libertadora (Dércoli, 2018). Por lo tanto, nuevamente ¿Qué es lo que ocurrió? ¿En base a qué experiencias se pensaron estos procesos? ¿Surgieron de la nada? ¿Sobre qué antecedentes buscaban construir su legitimidad?

La revista 4161, seguramente por su brevedad, no pueda darnos respuestas concluyentes, pero tal vez nos sea de utilidad para comprenderla como elemento de la construcción de una cultura política universitaria del peronismo. Cultura política que como construcción es clave en la interpretación de las experiencias y en la construcción de legitimidad. De este proceso también forman parte por intelectuales (4161 se referencia con algunos de ellos) como Hernández Arregui, Jauretche, José María Rosa; podemos pensar a estos intelectuales y sus obras como los mediadores entre el período de gobierno y la etapa de la proscripción, la idea de cultura política es de utilidad ya que nos permite pensar esta continuidad. Entendemos por cultura política una construcción discursiva e identitaria fuente unificación de representaciones que unifican criterios para la acción colectiva.

\section{Los principales rasgos}

La revista tomaba por nombre el número del decreto que proscribía al peronismo, de esa manera ellos convertían en elemento positivo aquella marca negativa de la Libertadora. Desconocemos la cantidad de ejemplares que se imprimieron; sin embargo, en la editorial del segundo número los autores se regocijaban de la buena recepción y sostenían que muchos de los temas surgieron de aportes de lectores.

Question, Vol. 1, N. ${ }^{\circ}$ 63, julio-septiembre 2019. ISSN 1669-6581

Instituto de Investigaciones en Comunicación | Facultad de Periodismo y Comunicación Social | Universidad Nacional de La Plata La Plata | Buenos Aires | Argentina

Página 8 de 18 
Uno de los rasgos centrales que se destaca es la caracterización de la "la Universidad [como] un instrumento de colonización mental y tergiversación ideológica, al servicio de los intereses oligárquicos e imperialistas" $(4161,1963)$. Esta definición distanciaba a la JUP del reformismo universitario, ya que este se había enrolado en el "mero gremialismo" "circunscripto a los límites del régimen legal universitario"; que había llevado a "falsa opción Reforma-HumanismoCentrismo". Según ellos, la verdadera alternativa estaba en "que no hay ni puede haber liberación social del pueblo sin liberación nacional de la patria, no puede haber una universidad al servicio de la Nación y del pueblo argentino sin una previa y total transformación de las estructuras económico, políticas y sociales" $(4161,1963)$. De forma tal que podemos afirmar que la JUP afinca su identidad política y su acción en relación directa a la comprensión de la función social de la universidad como "instrumento" de la dependencia. Para la revista, la Argentina era un país dependiente, por lo tanto "la Universidad ha sido y es, en lo ideológico, expresión correlativa de las instituciones económicas, políticas, culturales vigentes en el país" (4161, 1963). En ella se retoma la caracterización de la universidad como institución condicionada por una determinada historia nacional, por un lugar concreto en sistema mundo. Aquí podemos observar la conexión con las intelectuales que mencionamos. Por ejemplo, Hernández Arregui (2014) afirmará que: "La historia de nuestra Universidad es, por eso, la historia de nuestra oligarquía... Esa Universidad, sin ritmo ni estilo peculiar, fue el medio más sutil del predominio espiritual del coloniaje" (2014: 73); idea que también aparece en la revista y que es el título del artículo central del número uno: "La Universidad colonizada".

Como vimos, la conclusión fue entonces que no podía existir otra universidad si no había un proyecto alternativo de Nación. Universidad y proyecto de país se retroalimentarán entre sí, por lo tanto concluirán que "el peronismo requería una transformación más profunda de la Universidad, que la convirtiera en órgano de expresión de la conciencia de soberanía y nacionalidad que emergía en el país" $(4161,1963)$. Esto constituía una diferencia con reformismo-cientificismo que proyectará su universidad en relación a la definición de autonomía en referencia negativa hacia el Estado y la libertad institucional para la auto organización interna, según lo que expresa la revista.

La concepción de articulación intrínseca entre proyecto de nación y misión de la universidad aparece en diferentes artículos. Este esquema será utilizado para explicar las posiciones contrarias de la universidad a los gobiernos populares. Podemos advertir el siguiente esquema: durante el primer peronismo se buscó poner a la universidad en función de las demandas del crecimiento de la nación y la universidad por su mentalidad colonial prestó una férrea oposición; en cambio, a partir de 1955 con la vuelta de un proyecto de dependencia a la nación, se asiste a un proceso de intromisión de intereses extranjeros mediante el 
financiamiento internacional y los universitarios, mientras formalmente se "respete" su autonomía apoyan completamente. A partir de lo anterior, se explica la asociación que la revista denuncia entre reformismo y proyecto de universidad oligárquica, debido a que el reformismo aparecerá como actor central de las medidas limitacionistas y del direccionamiento que los créditos del extranjero realizaban sobre la universidad $(4161,1964)$. Más aún, en otro artículo, los intelectuales colonizados y universitarios serán asociados a un entramado que incluye a grandes capitales nacionales y extranjeros, asociados a EE.UU., y que esto los constituye en "enemigos internos" enquistados en nuestras estructuras económicas, educacionales, artísticas, política (4161, 1964: 3).

Debemos advertir que la revista subvalora las diferencias entre reformistas, humanistas, comunistas y cientificistas; siendo todas identidades que ellos sintetizaban como diferentes facetas de la universidad colonizada, y que estaban asociadas a la idea de universidad como privilegio, que en definitiva era consecuencia de la estructura económica y social; según ellos esa era la razón de "los permanentes desencuentros históricos de los estudiantes con las masas populares" $(4161,1963)$. Al mismo tiempo, esa universidad colonizada se expresaba, según ellos, en "las propias concepciones político-universitarias vigentes en las organizaciones estudiantiles facilitan una visión unilateral del proceso histórico, y a través de una lucha parcializada y gradual por conquistas 'gremiales' desarraigan a los universitarios de la lucha integral del pueblo argentina" $(4161,1963)$.

Los artículos vislumbran un cambio en el estudiantado, lo muestran signado por la decepción y en vías de transformación de su mirada sobre los problemas políticos. Allí radicaba para la JUP uno de sus ejes de intervención: el peronismo debía aprovechar y darse a la conquista de estos sectores que ahora miraban al peronismo con otros ojos. Pero recalcaban que no había que olvidarse que el estudiantado era "un estamento altamente sensible de las clases medias", sectores propensos a "degenerar en conflicto estériles y absurdos" $(4161,1963)$; para ellos, la JUP no debía caer en eso, debía articular a la universidad con la lucha del pueblo, ella debía dar la lucha en la universidad pero sin caer en las disputas intestinas de la propia institución, que no conducían nada. Concretamente, según esa mirada la transformación de la universidad en sí era absurda, la universidad sólo podía ser transformada por un proceso político nacional que la excede, ya que la universidad era "una institución aristocrática de elites, al servicio de la estrategia cultural de la oligarquía y los imperialismo. $Y$ ello, no se puede arreglar 'incorporando activamente a los estudiantes en el consejo directivo'...aunque no se debe desechar absolutamente esta táctica dentro de una actividad mucho más amplia y en otros niveles dirigida hacia los grandes fines, la participación del estudiantado en la lucha de liberación" (4161, 1964: 6).

Question, Vol. 1, N. 63 , julio-septiembre 2019. ISSN 1669-6581

Instituto de Investigaciones en Comunicación | Facultad de Periodismo y Comunicación Social | Universidad Nacional de La Plata La Plata | Buenos Aires | Argentina

Página 10 de 18 


\section{El sentido del peronismo en la universidad}

Los anteriores elementos aportan en la configuración de lo que llamamos una cultura política universitaria del peronismo, ya que nos permiten entender las representaciones construidas en torno al sentido de la lucha política que el peronismo debía dar en la universidad; mencionemos dos rasgos recurrentes: primero el de lo colonizado, representando por la historia de la universidad, el cientificismo, el reformismo y, segundo, la necesaria articulación entre lucha política en la universidad y la cuestión nacional.

Del mismo modo, aparecen otros elementos que también ilustran ciertos rasgos de vanguardismo. Concretamente, en los momentos en que ellos se sitúan en el lugar de protagonistas en el proceso de "unir las inquietudes, problemas, reivindicaciones y luchas estudiantiles con el proceso político protagonizado por las masas" $(4161,1964: 6)$. Ellos se ubican como parte del estudiantado y del pueblo, aun así no dejaban al mismo tiempo de pensarse como una vanguardia en este proceso. Más aún, se perciben como actores esclarecidos con una misión: "destruir los prejuicios antiperonistas, promover la 'nacionalización' y definición revolucionaria de los universitarios" (1964: 6). Lo anterior convivía con el reconocimiento constante del liderazgo de Perón y al pueblo como actor central del proceso de liberación.

Debemos destacar una particularidad, en los artículos cuando se concibe la propia condición de universitarios, y de futuros egresados, no aparece la representación del intelectual sino la del profesional-trabajador. La siguiente cita lo ilustra muy bien:

\footnotetext{
Debemos hacer conciencia que como futuros profesionales, estamos vitalmente interesados en los objetivos de soberanía nacional, desarrollo industrial independiente y justicia social, que crearán amplias posibilidades de trabajo profesional sin dependencia de las fuerzas privilegiadas $(4161,1963)$.
}

Podemos concluir, además, que hay una conexión entre el universitario y la nación a partir de su futura proyección laboral. En otras palabras, el horizonte en el que se piensan es en de un trabajador, con capacidad de desarrollarse en una comunidad que sólo se puede desarrollar en virtud de un proceso de liberación. Esto le da una singularidad a la revista ya que pone en el centro el carácter "integral" en el cual la JUP planteaba la lucha universitaria, no exclusivamente como reivindicación gremial del estudiante sino del universitario como trabajador y como integrante de una nación. Esta era la forma, según ellos, de romper con la histórica oposición del estudiante universitario a los gobiernos populares: por un lado a partir de 
clarificar la unidad de intereses y, por otro, a partir de la reivindicación del universitario como parte del pueblo. Al mismo tiempo que la organización debía construir un pensamiento nacional que era deliberadamente excluido de la universidad.

\section{La invención de la tradición}

El estudio de la revista nos muestra el proceso de delimitación de un adversario para el peronismo de la universidad, aquel será nombrado como reformismo, desarrollismo, humanismo o cientificismo. No importa que no haya una definición acabada de los mismos, ya que estos conceptos cumplen indistintamente la función de representación del adversario de la JUP. En líneas generales la revista esboza algunas de las características de sus contrincantes: los identifica como aquellos que piensan la universidad de forma aislada, como cosa en sí y escindida del destino de la Nación; a esto se suma que se le endilga ser aquellos que terminan afirmando que la autonomía constituye un resguardo suficiente para garantizar el pleno desenvolvimiento de la actividad universitaria. Hecho grave, según la visión de 4161, debido a que la separación de los destinos de la Nación y de la Universidad tiene consecuencias importantes, particularmente impide, a los reformistas, humanistas, etcétera, observar la dimensión de condicionamiento político que implica los incentivos a la investigación que financia el capital extranjero. A pesar de destacar la existencia de matices al interior del reformismo, reconociendo la presencia de un sector de izquierda, con todo no ven en ellos un actor con potencialidad transformadora, pues están formados en una matriz de pensamiento liberal; razón por la que finalizarán abrevando en la filas del antiperonismo y en la perpetuación de debates intrascendentes. Por todos esto, de acuerdo a la Revista, estos sectores de izquierda del reformismo terminan siendo parte de la reproducción de la universidad "isla". Aquí encontramos las críticas al reformismo universitario que luego también realizarán revistas como Envido o Antropología del Tercer Mundo (Cfr. Recalde, 2016; Dip, 2017). Cabe destacar, que estas mismas críticas a las derivas de la Reforma, o sea al reformismo, están presentes en las reflexiones sobre la universidad que realizan pensadores del campo nacional, más específicamente, Jauretche se refiere críticamente a la Reforma en varias ocasiones, a pesar de reconocerle en sus inicio un impulso transformador (2008: 138).

Desde nuestra visión, el reformismo universitario es un recorte selectivo de la Reforma con el fin de nutrir la construcción de una identidad política, en particular en el reformismo desaparecen el antiimperialismo y el latinomericanismo. Aunque también destaquemos que sobre estos elementos el peronismo armará la imagen en la que inscribe a su adversario. Junto 
con esto aparece la crítica a la idea de autonomía como reducida a un aspecto de carácter formal y limitada en identificar como factores de heteronomía el Estado y la política; lo que llamamos una interpretación liberal, ya que la autonomía como sinónimo de no interferencia por parte del Estado. Obviamente, esta limitación deja afuera otros aspectos de heteronomía como la dependencia cultural y los intereses del mercado, aspectos centrales que configuraban la crítica al modo civilizatorio de los países centrales. Sobre algunos de estos elementos el peronismo construirá su discurso para la universidad.

A esto debemos sumarle el concepto de invención de la tradición, este nos permite pensar la relación que se construyó entre cientificismo y Reforma, para entenderla como una construcción deliberada de los actores y de la historiografía. En el caso de esta última, ambos términos aparecen unidos, el período que aquí estudiamos es consagrado por estos relatos como "la época de oro" de la investigación y se asoció a la vuelta los principios reformistas como rectores de las universidades. Pero otros actores, como 4161, ven aquí un período de subordinación frente al imperialismo -lo cual entraría en contradicción con la Reforma si recordamos el contenido antiimperialista y antinorteamericano de la misma-, desde allí nace la crítica al cientificismo y su carácter peyorativo. Este aspecto aparece en la revista de la siguiente manera: "Las becas se distribuyen generosamente para ir estudiar al extranjero las especialidades que interesan al imperialismo" (4161, 1964). Para "denunciar" la conexión política entre investigación y orientación por medio del financiamiento, esto recuerda lo que años más tarde Varsavsky llamará colonialismo científico. Cabe recordar que la Reforma era también una denuncia al imperialismo cultural.

Para concluir, la invención de la tradición reformista es una operación historiográfica que fue parte del intento de los reformistas-cientificistas por conectar su accionar y legitimarlo con un pasado que los justificaba, legitimando así su identidad política. Sin dudas esto debe ser asociado a un recorte de los múltiples legados de la Reforma. Algo que más tarde se consolidó en versiones historiográficas que afirmamos entendieron el devenir de la Universidad tal como si la misma se tratará del despliegue de la razón reformista. En otras palabras, se construyó la Reforma como un modelo y sus "principios" en un tipo ideal; a partir de esto se juzgó la historia de nuestras universidades en función de cuánto más se alejaban o se acercaban a esa idea, convertida en un "modelo" de universidad reformista. Por lo tanto, a través de una asociación Reforma-democracia: cuanto más se acercaba a ese recorte el historiador dirá que la universidad vivió un momento de florecimiento. A modo de crítica, podríamos decir que los trabajos que subscriben a esta línea operan de forma similar que las simplificaciones revisionistas: ven la historia atravesada por un conflicto esencial que se desarrolla con particularidades en cada momento pero que responde a un mismo conflicto permanente. La 
consecuencia de la hegemonía reformista, o de la tradición reformista en tanto su autopostulación como la única organización democrática de la universidad, llevó a que cualquier intento por desarrollar una política para el sector deba pensarse en función de la esta interpretación limitada de la Reforma. En algún punto la idea de Reforma ocupa el lugar de telos en la historia de la Universidad, así como la Nación ocupa ese lugar en los relatos nacionales.

Continuemos con otro aspecto, la potencialidad de esta tradición inventada podemos observarlo en la revista 4161, ya que observamos que los artículos discuten no con lo Reforma, sino con aquel recorte; o sea discuten con la Reforma de los reformistas. Entonces apelan al cuestionamiento de la Reforma como hecho en esencia antipopular por su deriva opositora a los gobiernos populares o por la promoción de una universidad "isla"; cuando esto sería más bien algo achacable al reformismo como tradición inventada o recorte. De esta forma también la propia revista ayuda a consolidar, por oposición, a la propia tradición inventada, que consecuentemente le permite construir la identidad o "los objetivos peronistas en la universidad" (1964: 6).

La crítica al reformismo en esta clave es central en el desarrollo de lo que hemos llamado una cultura política universitaria peronista. Pero a esto se suman otros elementos que la configuran, por ejemplo, una concepción democrática de la universidad distinta; cuya particularidad remite en afirmar el ingreso de todos los sectores sociales y una función liberadora. En este sentido, existe la reivindicación del desarancelamiento de 1949 y las apuestas por la organización de la universidad desde el Estado en función de una política de soberanía. La revista ubicará estos planteos en la oposición al reformismo y propios de la obra del primer peronismo. A diferencia de esta política, el reformismo se concentrará exclusivamente en ampliar la participación institucional de los estudiantes en los órganos de cogobierno. Aunque, como lo hemos mencionado, la JUP no despreciaba esta última lucha sino que debía ser articulada a una dimensión política macro. Todos estos aspectos se suman a los planteos de la revista por asociar el carácter nacional de la universidad al abandono de un ideal colonizado. Para ello debía esta debía dejar de ser el aparato de la penetración de las ideologías y pedagogías del imperialismo. Entonces, 4161 aportó a la construcción de una cultura política universitaria que se asocia a las transformaciones universitarias del primer y segundo gobierno de Perón, pero que incorporó también elementos presentes en las críticas desarrolladas por Hernández Arregui y Jauretche ya entrados los años cincuenta. No obstante hay que desatacar que 4161 se mostrará crítica con algunos elementos de la política universitaria del primer peronismo, por ejemplo, la creación de la Confederación General Universitaria. 


\section{Comentarios finales}

Analizamos la revista 4161 con el objetivo de encontrar como evidencia, aunque acotada, el desarrollo de una cultura política que constituye al peronismo universitario. El concepto de cultura política nos permitió tejer un puente entre algunos elementos de la política universitaria del primer peronismo y la revista 4161, y aún queda pendiente indagar su relación con lo que más tarde será la masificación del peronismo universitario a principios de los setenta. El concepto de cultura política nos fue de utilidad para pensar esta continuidad desde las ideas y conceptos, ya que no puede ser afirmado desde las personas que escribieron en la revista, por la sencilla razón de que ninguna siguió en contacto con el mundo universitario posterior; aunque si con el mundo de la militancia peronista de fines de los sesenta y principios de los setenta.

Pudimos observar la relación entre la cultura política universitaria del peronismo y la reformista, lo cual nos da indicios de un intento de sincretismo, de incorporación y no de exclusión absoluta, de un diálogo entre pasado, presente y porvenir pero como "fenómeno colectivo, compartido por grupos enteros que dicen tener los mismos postulados y que han vivido las mismas experiencias" (Berstein, 1997: 403). Aspecto que queda señalado en la revista: "las filas de JUP engrosan con jóvenes provenientes de diversos campos ideológicos, desde el fubismo al falangismo, incluyendo humanistas e independientes" $(4161,1963)$. En otras palabras, no estamos ante una invención arbitraria sino, ante un proceso de resignificación de la universidad y de los universitarios en el marco de una etapa que según estos protagonistas se encuentra atravesada por el clivaje dependencia-soberanía. Si, siguiendo a Terán, la política era la esfera que otorgaba sentido a todas la demás actividades en este período, nuestro aporte es que a esto habría que agregarle que no por ello se perdió de vista la especificidad de la universidad, más bien del diálogo entre la política y esta, surgió una cultura política particular que no la habría anulado como instancia de lucha sino que la situó en la disputa política, atravesada por los mismo clivajes que la nación. $Y$ aquí se abre un interrogante a futuro ¿se politizó la pelea universitaria sin que perdiera su especificidad? Preliminarmente, creemos que la revista fue un intento de eso.

Además, vimos cómo algunos sectores del peronismo pensaron la militancia en la universidad en tensión con la tradición del reformismo universitario, pero también un diálogo con determinados sectores del mismo que se encuentran en proceso de "nacionalización". A nuestro juicio, la cultura política que emergerá se apropiará de elementos del reformismo, ya que hemos advertido un diálogo entre reformismo y peronismo, en tanto la JUP no descarta la lucha institucional dentro de la universidad, digamos la lucha electoral por las posiciones en los 
órganos de cogobierno. Más bien se crítica al reformismo porque ésta es su única ambición y cree que la universidad puede transformarse sobre sí. Lo que distingue a la JUP entonces será que sin desmerecer la militancia universitaria afirmará que su potencial transformador surgiría de articular esa lucha (institucional, electoral por los cogobierno, etcétera) en una perspectiva política más amplia. El impacto del reformismo en la cultura política universitaria del peronismo puede verse en el cambio con respecto a la mirada que había sobre los órganos de cogobierno en las leyes. Por ejemplo, en la de los primeros gobiernos hay una visión absolutamente crítica de los mismos, pero que luego en la Ley Taiana de 1974 se modificará incorporando estos elementos de la "tradición" reformista al cuerpo legal y es más ampliando la participación a los trabajadores nodocentes. Esto no permite fortalecer la idea de una cultura política que se nutre y transforma, que se encuentra en diálogo (Berstein, 1997: 398), razón por la cual nos alejamos de los esencialismos que muchas veces nos plantean la idea de las tradiciones centradas en sí mismas, esta perspectiva nos alerta sobre los diálogos y los múltiples aportes que abrevan en los procesos de construcción de identidades políticas. Por último, si bien se trata de un trabajo preliminar y de una exploración podemos ver que esta perspectiva nos permite matizar las interpretaciones que afirman al movimiento de "nacionalización" como intempestivo o demasiado sujeto a causas contingentes. Siguiendo con la distinción de Bloch entre causas y condiciones (2015), podemos observar que efectivamente existen causas contingentes, pero la revista y el concepto de cultura política nos permiten pensarlas la existencia de condiciones, de mayor permanencia, de orden más estable que una causa inmediata, para que ese proceso derivara en una forma particular y no en cualquier otra. En otras palabras, esta cultura política será parte de la matriz que marcará a la juventud universitaria de los años setenta y estará como base de los proyectos de transformación institucional. En función de esta continuidad de problemas es que podemos, siguiendo a Gilman (2012), hablar de los 60 y los 70 como época, como unidad "sesenta/setenta". En el campo particular de la universidad o del campo intelectual podríamos pensar como rasgos de unidad la idea de un conocimiento tensionado por determinaciones políticas, es decir entre la soberanía y la dependencia. Las instituciones universitarias como reproductoras de la dominación, la predica de un intensa politización, y no la neutralidad y la autonomía, como claves de una nueva "verdad" del conocimiento, que es antes que nada transformación y no descubrimiento de lo dado.

\section{Notas}

(1) Hugo Chumbita fue uno de los impulsores de la revista, lo entrevistamos en mayo de 2018.

Question, Vol. 1, N. ${ }^{\circ}$ 63, julio-septiembre 2019. ISSN 1669-6581

Instituto de Investigaciones en Comunicación | Facultad de Periodismo y Comunicación Social | Universidad Nacional de La Plata La Plata | Buenos Aires | Argentina 


\section{Bibliografía}

Altamirano, C. (2013). Peronismo y cultura de izquierda. Argentina: Siglo XXI.

Barletta, A. M. (2001). La "peronización" de los universitarios (1966-1973). Elementos para rastrear la constitución de una política universitaria peronista. Pensamiento Universitario, 9, 1-14.

Berstein, S. (1998). La cultura política. En Rioux, J. P. y Sirinelli, J. F. (dirs.). Por una historia cultural (pp. 389-405). México: Taurus.

Buchbinder, P. (2011). Historia de las Universidades Argentinas. Buenos Aires: Sudamericana.

Bloch, M. (2015). Introducción a la Historia. México: FCE.

Dércoli, J. (2014). La política universitaria del primer peronimo. Buenos Aires: Punto de Encuentro.

Dércoli, J. (2018). La Reforma Universitaria, el reformismo y las transformaciones universitarias del peronismo. En Rinesi, E.; Pelusa, N. y Ríos, L. (Comps.). Las libertades que faltan. Dimensiones latinoamericanas y legados democráticos de la Reforma Universitaria de 1918 (pp. 171-190). Buenos Aires: UNGS.

Dip, N. (2012). La universidad en un mundo de tensiones. Una aproximación al itinerario político-universitario de las organizaciones de estudiantes y docentes peronistas de los años sesenta a través del estudio del proyecto de Universidad Nacional-Popular propuesto en la revista Envido. VII Jornadas de Sociología de la UNLP, Departamento de Sociología de la Facultad de Humanidades y Ciencias de la Educación, La Plata.

Dip, N. (2017). Libros y alpargatas. La peronización de estudiantes docentes e intelectuales de la UBA (1966-1974). Buenos Aires: Prohistoria.

Friedemann, S. (2017). De las Cátedras Nacionales (1967-1971) a la Universidad Nacional y Popular de Buenos Aires (1973-1974). Experiencias configuradoras de institucionalidad universitaria. Sociohistórica, $39 . \quad$ Recuperado de https://doi.org/https://doi.org/10.24215/18521606e026

Faierman, F. (2017). Ciencia Nueva: de revista de ciencia y tecnología a revista de vanguardia. Cuadernos Del CEL, 2(4), 14-26.

Gilman, C. (2012). Entre la pluma y el fusil debate y dilemas del escritor revolucionario en América Latina. Buenos Aires: Siglo XXI.

Jauretche, A. (2008). Los profetas del odio y la yapa. Buenos Aires: El Corregidor.

Hernández Arregui, J. J. (2014). La formación de la conciencia nacional. Buenos Aires: Peña Lillo-Ediciones Continente. 
Hobsbawm, E. (1983). Introducción: La invención de la tradición. En Hobsbawm, E. y Ranger, T. (eds.). La invención de la tradición. Barcelona: Crítica.

Neiburg, F. (1998). Los intelectuales y la invención del peronismo: estudios de antropología social y cultural. Buenos Aires: Alianza.

Recalde, A. (2016). Intelectuales, peronismo y universidad. Buenos Aires: Punto de Encuentro.

Recalde, A. y Recalde, I. (2007). Universidad y liberación nacional. Buenos Aires: Nuevos Tiempos.

4161 (octubre de 1963). Órgano de la Juventud Universitaria Peronista, 1(1).

4161 (junio de 1964). Órgano de la Juventud Universitaria Peronista, 2(2).

Sarlo, B. (1992). Intelectuales y revistas: razones de una práctica. En América. Cahiers du Criccal, 9(10). Le discours culturel dans les revues latino-américaines, 1940-1970, pp. 9-16. doi: 10.3406/ameri.1992.1047

Sigal, S. (2002). Intelectuales y poder en Argentina. La década del sesenta. Buenos Aires: Siglo XXI.

Terán, O. (2013). Nuestros años sesentas. La formación de la nueva izquierda intelectual argentina. Buenos Aires: Siglo XXI.

Williams, R. (2009). Marxismo y literatura. Buenos Aires: Las Cuarenta. 